dissemination patterns. Tumour biopsies were collected (range 4-15, median 9), placed in short-term cultures, treated with

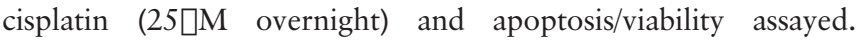
When relapsed, patients also had paired biopsies collected for genomic and phenotypic analysis. DNA was extracted from tumours ( 5 per patient, $n=49$ patients plus relapse samples) and Illumina Human OmniExpress genotyping performed. Allele-specific copy number $(\mathrm{CN})$ was quantified using ASCAT. Genomic heterogeneity was quantified as the estimated number of $\mathrm{CN}$ aberration events distinct between each pair of tumour deposits. Clonal diversity within a patient's deposits was calculated using the difference between within-patient and between-patient heterogeneity.

Results Broad heterogeneity was detected in response to platinum treatment across cases at the phenotypic level in vitro $(n=49)$, with higher variances in apoptosis induction observed in patients with platinum resistant disease. Genomic analysis revealed widespread variations in patterns of evolution for different patients' tumours, including the relationship between primary tumours and relapsed disease. Extensive variations in CCNE1, MYC and PTEN CN were observed across multiple tumours in the same patients, and overall higher CCNE1 CN associated with poorer patient outcome $(\mathrm{p}=0.038)$.

Conclusion Vast intra-tumoural heterogeneity is observed at the phenotypic and genomic level in HGSOC patients. Extensive copy number variations in genes such as CCNE1, MYC and PTEN across multiple disseminated samples within patients, indicates that sampling of a single tumour site does not accurately represent overall disseminated HGSOC biology and has implications for overinterpretation of studies relating to outcome and platinum-resistance.

Disclosures CF: advisory boards and honoraria from Roche, Tesaro, Sequana, Olympus, Astra Zeneca. Other authors have no disclosures to declare.

\section{NOVEL 3D MODEL SYSTEMS TO ASSESS HETEROGENEITY IN RESPONSE TO PLATINUM THERAPY IN HIGH GRADE SEROUS OVARIAN CANCER}

Jennifer Ploski, Katherine Nixon, Nikita Demchenko, Paula Cunnea, Christina Fotopoulou. Imperial College London; Division of Cancer; Department of Surgery and Cancer

\subsection{6/ijgc-2020-ESG0.172}

Introduction/Background High-grade serous ovarian cancer (HGSOC) is the most common subtype of ovarian cancer, characterised by vast genomic instability and heterogeneity and acquired resistance to platinum-based chemotherapy. However, matching the most beneficial treatment options to patients is difficult to predict due to different platinum resistance mechanisms and limited effective predictive biomarkers. A study characterising intra-tumoural heterogeneity in HGSOC has identified variations in phenotypic responses to platinum treatment between different metastatic sites. In this study, we aim to develop novel clinically-relevant 3D exvivo models of HGSOC to investigate the effect of the local microenvironment on metastatic tumour cells' response to treatment, and potential use as a screening tool to predict drug responses.

Methodology Three different ex-vivo models were developed: organotypic, organoid and tumour slice. For organotypic and organoid models, tumour cells were extracted from metastatic deposits obtained from defined anatomical regions during upfront radical debulking surgery of advanced stage HGSOC patients. Organotypic models were assembled using normal omental stromal cells embedded in Collagen-1 and tumour cells were added. Organoid models were propagated from tumour cells and embedded in basement membrane extract. For slice culture models, tumours were sliced into $350 \square \mathrm{m}$ sections using a vibratome and cultured on cell culture inserts. All models were treated with cisplatin and assessed for apoptosis and viability readouts.

Results Organotypic models showed that tumour cells cultured in $3 \mathrm{D}$ showed heterogeneity in response to cisplatin treatment, data showed a trend towards reduced response to treatment within $3 \mathrm{D}$ models compared to $2 \mathrm{D}(\mathrm{n}=8)$. Changes in patterns of response to treatment between samples from $2 \mathrm{D}$ to $3 \mathrm{D}$ within the same patient was also demonstrated $(n=5)$. Organoid models were successfully propagated from different metastatic sites and maintained long term growth (>15 passages). Histological read-outs for slice culture models demonstrated slices from different metastatic sites maintained viability in culture for up to 5 days.

Conclusion We have established growth, drug treatment conditions and assay read-outs for 3 different ex-vivo models of metastatic HGSOC. We have established that organoid culture must be generated within 24hours of tumour cell extraction. Furthermore, both fresh and viably frozen tumours can be used to generate organotypic and organoid models. The broader implication of establishing clinically-relevant complex tumour models as routine methodologies for screening novel therapeutics and capturing the complex heterogeneity of individual patients, may lead to better development of therapeutic strategies including tumour/microenvironment combination strategies and also better personalisation of therapy for patients with HGSOC.

Disclosures CF: advisory boards and honoraria from Roche, Tesaro, Sequana, Olympus, Astra Zeneca. Other authors have no disclosures to declare.

\section{Trophoblastic diseases}

\section{A CLINICAL AUDIT OF MOLAR PREGNANCIES AND GESTATIONAL TROPHOBLASTIC NEOPLASIA CASES OVER 1YR IN A TERTIARY CARE HOSPITAL OF EASTERN INDIA WITH RESPECT TO THE INCIDENCE OF DISEASE, FACTORS RELATED TO ETIOPATHOGENESIS, DIAGNOSIS AND MANAGEMENT}

Swarnabindu Banerjee. Medical College Kolkata; Medical Oncology

\subsection{6/ijgc-2020-ESG0.173}

Introduction/Background A Clinical Audit of molar pregnancies and gestational trophoblastic neoplasia cases over $1 \mathrm{yr}$ was conducted at Kolkata Medical College \& Hospital, a tertiary care hospital of Eastern India with respect to the incidence of disease, factors related to etiopathogenesis, diagnosis and management and effects on maternal morbidity and mortality.

Methodology A total of 10000 patients attended this institution during 2017 june to 2018 june for pregnancy or its complication in department of gynaecology \& obstetrics.85 molar 